\title{
Cyclic Olefin Resist Polymers and Polymerizations for Improved Etch Resistance
}

\author{
R. D. Allen, J. Opit, H. Ito, T. I. Wallow, D. V. Casmier, C. E. Larson, R. Sooriyakumaran, \\ D. C. Hofer, and P. R. Varanasi* \\ IBM Research Division, Almaden Research Center \\ 650 Harry Road, San Jose, CA USA 95120 \\ *IBM Microelectronics Division, East Fishkill Facility \\ 5800 Route 52, Hopewell Junction, NY USA 12533
}

This paper will describe the strengths and unique challenges of building $193 \mathrm{~nm}$ resists from acrylic polymers and cyclic olefin polymers. In particular, cyclic olefin (alternating) copolymer synthesis and properties will be discussed.

Keywords: Cyclic olefin polymers, free radical polymerization, alternating polymers, dissolution properties, etch resistance

1. Introduction

Since the early days in the development of new materials for $193 \mathrm{~nm}$ single layer resists, the recognition that a successful materials platform would combine high transparency with robust etch resistance has been a universal theme of most investigations.[1] The target has been the search for a polymer material family which behaves at $193 \mathrm{~nm}$ as the hydroxystyrene polymers do at $248 \mathrm{~nm}$ (dissolution control, transparency and etch resistance). Early in the development of 193 resist materials, the polymer platform of choice was the acrylic polymer backbone because of its straightforward polymer synthesis, transparency and seeming flexibility for resist design.[1,2] However, despite the early success at excellent lithographic performance of acrylic 193 nm photoresists,[2] and reasonably successful efforts to improve the plasma etch resistance of these materials, $[3]$ the etch resistance of these materials appears to have limits that make them significantly less robust than the classic poly(hydroxystyrene)-based $248 \mathrm{~nm}$ resist systems.[4]
Through recent extensions of DUV (248nm) photoresists, the advent of high NA (ca. 0.7) exposure tools and optical enhancement techniques, lithography processes to $0.18 \mu$ and even $0.15 \mu$ features have recently been demonstrated.[5] It seems clear that the introduction of $193 \mathrm{~nm}$ lithography into semiconductor manufacturing will be at feature sizes at or below $0.15 \mu$ (for nested features). As a result, resist film thicknesses must necessarily decrease, placing more emphasis on the improvement of etch resistance of $193 \mathrm{~nm}$ resists beyond the demonstrated values for acrylic systems.

Polymer and resist research efforts throughout the world have endeavored to identify promising new $193 \mathrm{~nm}$ resist-compatible polymer chemistries with the result that a number of new chemical platforms have emerged as candidates, including free radically polymerized alternating copolymers of cyclic olefins with maleic anhydride,[6] metal-catalyzed addition polymerization of cyclic olefins, [7] metal-catalyzed ring opening metathesis 
polymerization (ROMP) of cyclic olefins,[8] and cyclopolymerized materials.[9]

Each of these polymer families represents a new first application of an (often unproven) polymerization technique to photoresist materials. The ultimate utility of each of these polymers and polymerization chemistries for photoresist applications will depend on the control of the polymerization, flexibility in monomer and polymer synthesis, chemical functional group tolerance of the polymerization technique and compatibility of resultant polymer properties with photoresist design and formulation requirements. This paper will attempt to compare and contrast the free radical polymerizations and the derived polymer materials from two of the major candidates, acrylic and cyclic olefin alternating copolymers.

\section{Results and Discussion}

\subsection{Acrylic Resists}

An attractive route for the synthesis of polymers for resist applications is the preparation of acrylic polymers. This route is quite possibly superior to the synthesis pathway(s) for hydroxystyrene-based polymers for DUV lithography. Acrylic polymers can be prepared using a number of polymerization mechanisms (e.g., free radical and a number of "liiving" polymerizations) all of which offer clean, high yielding preparations, with good molecular weight control.

The etch resistance of these materials is somewhat dependent on etch process (e.g., polysilicon vs. oxide etch) with encouraging results in polysilicon processes and discouraging results in oxide etch.[4]

The imaging performance of acrylic resists is good and steadily improving. For example, the 1997-era IBM V2 resist (Figure 1) has demonstrated high resolution with good process latitude (but uses a diluted developer).[10] We have recently investigated the use of resolution enhancement techniques for $193 \mathrm{~nm}$ resist processes using alternating phase shifting masks on a ISI ArF Microstep (0.6NA, sigma $=$
0.3). Process window studies for nested features down to $90 \mathrm{~nm}$ revealed that substantial dose and focus latitude exist at 90 and $100 \mathrm{~nm}$, while opening up the space between lines slightly (1:1.5 to 1:2.5) produced good latitude down to and including $70 \mathrm{~nm}$ features. Figure 2 shows the process window for $100 \mathrm{~nm}$ nested lines using PSM with an acrylic (Version 2) resist.

In summary, alicyclic-modified acrylic resists may well serve as a first generation $193 \mathrm{~nm}$ technology. These systems can provide excellent imaging performance coupled with reasonable etch rates in polysilicon processes. Problems with durability in oxide etch processes could possibly limit the use of these materials in the future, however.
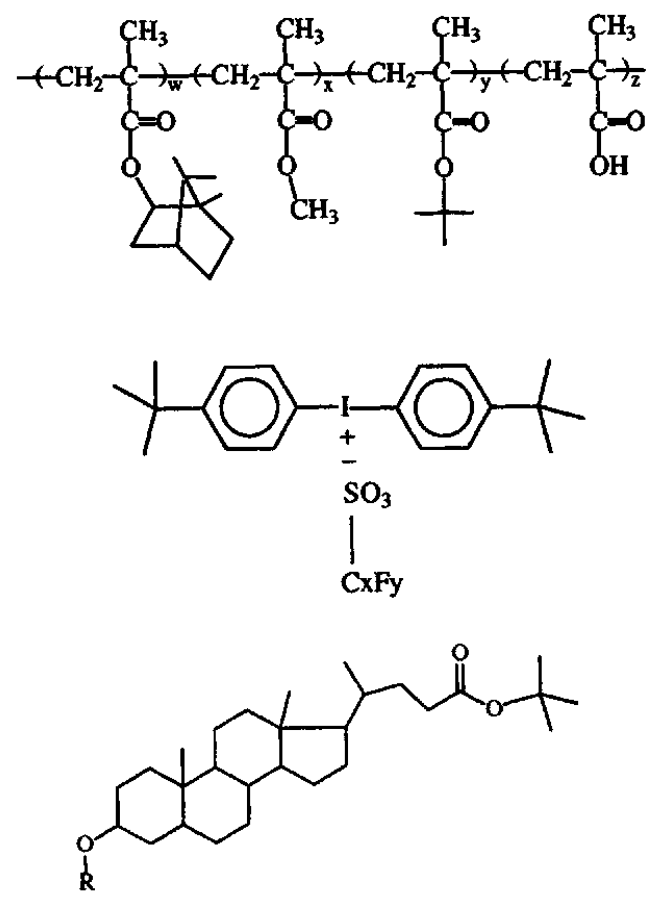

Figure 1: Chemical structures of components of IBM V2 Resist

\subsection{Cyclic Olefin-Maleic Anhydride}

Alternating Polymers Free radical polymerization of norbornene and its derivatives is not practical due to low reactivity. The use of maleic anhydride as a comonomer (at levels of approximately $50 \mathrm{~mole} \%$ ) transforms this sluggish polymerization into a substantially more efficient synthesis.[11] We examined the synthesis of cyclic olefin polymers via these alternating 
copolymerizations and the properties of the resulting materials. These findings will be presented below.

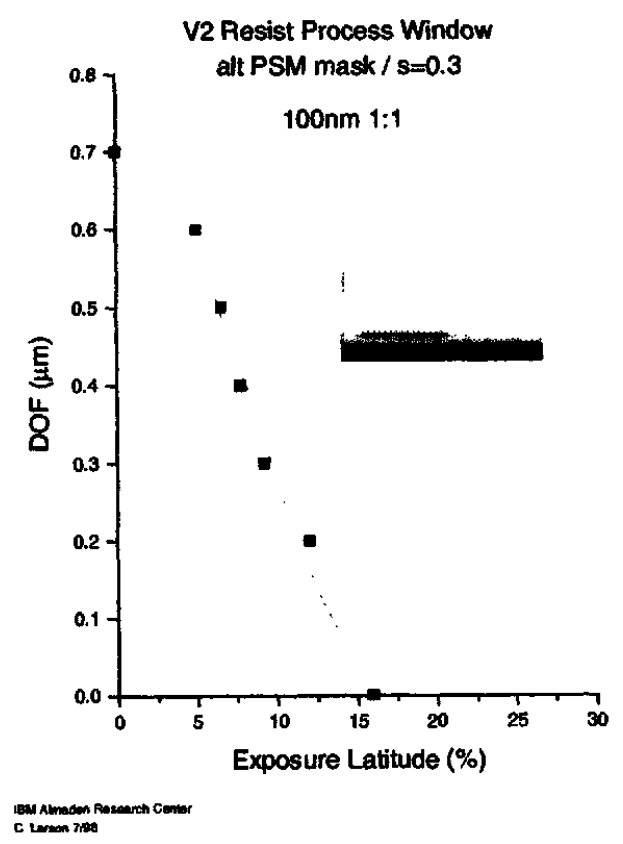

Figure 2: Process window for $100 \mathrm{~nm}$ nested features using an acrylic resist (IBM Version 2) exposed at $193 \mathrm{~nm}$ using an alternating phase shifting mask

Initially, the monomers used for this investigation were norbornene (unsubstituted) and maleic anhydride. The copolymerizations were run using conventional solvents (THF, Dioxane) and initiators (AIBN). The collected yields and molecular weights for several of these polymerizations are shown in Table 1 . The data clearly show a number of interesting trends. The yields range from moderate to high $(75-90 \%)$, not all that different from traditional methacrylate polymerizations. The departure from normality comes with regard to molecular weight.

Table 1: Free Radical Copolymerization of Norbomene and Maleic Anhydride

\begin{tabular}{|c|c|c|}
\hline Polymerization & $\begin{array}{c}\text { Percent } \\
\text { Yield }\end{array}$ & Mw \\
\hline AIBN/THF & 91 & 1,970 \\
\hline AIBN/Dioxane & 75 & 3,900 \\
\hline $\begin{array}{c}\text { AIBN/Dioxane/ } \\
\text { Thiol }\end{array}$ & 75 & 3,200 \\
\hline
\end{tabular}

Molecular weights are low compared to most free radical polymerizations. They are also strangely insensitive to initiator concentration. The data in the table represents an initiator concentration of 4 mole percent. Running the copolymerization at initiator concentrations from 2 to 6 mole percent makes very little difference in the molecular weight. Typically, in free radical polymerization of acrylic monomers, as the initiator concentration is increased, the molecular weight decreases, due to the increased radical concentration. This high radical flux increases the possibility that the free radicals at the polymer chain-ends can "find eachother" a necessary condition for termination. For this reason, polymer chains generated at high conversions (in more typical polymerizations) have a higher molecular weight, as the viscosity inhibits diffusion of radical chain-ends, sharply lowering the termination rate, but leaving the propagation rate (rate of polymerization) largely unaffected (monomer diffuses easily regardless of viscosity). This gel effect, also known as the Tromsdorff effect,[12] leads to the formation of a high molecular weight tail, increasing the polydispersity to greater levels than theory predicts $(1.5-2.0)$. The molecular weight of the norbornene alternating polymers (low MW and relatively narrow polydispersity) point to the absence of the gel (Tromsdorff) effect (at least in the monomer concentration range employed in this study (25\%)). This is presumably due to the stiff, highly extended nature of these polymers in solution, exposing the free radical at the chain end to termination. An alternative (and possibly complementary) explanation is that in these alternating polymerizations, the propagation rate is (relatively) low and the termination rate (relatively) high. The high termination rate makes it difficult to control the polymerization to such a point that one can "push" the molecular weight significantly higher. Interestingly, at higher polymerization temperatures (ca. $100{ }^{\circ} \mathrm{C}$, refluxing dioxane) the molecular weight does in fact increase somewhat, suggesting that the propagation rate may be increased relative to 
termination.

The properties of these "simple" copolymers (see Table 2) provide some unsettling information. First, the glass transition temperature $(\mathrm{Tg})$ is a strong function of the molecular weight. This is not surprising, in that these copolymers are at the low molecular weight end of the spectrum. A fine example of a material with tremendous molecular weight sensitivity are novolak polymers, another material of very low molecular weight, further complicated by high polydispersity.[13] This severe molecular weight influence on $\mathrm{Tg}$ suggest other properties (dissolution rates, photospeeds) may also be strongly influenced by changing molecular weights at this end of the spectrum. This can be an important variable in batch-to-batch variation in this class of materials.

Table 2: Properties of Norbornene-Maleic Anhydride Alternating Copolymers

\begin{tabular}{|c|c|c|c|}
\hline Polymer & Mw & Tg & $\begin{array}{c}\text { Abs/um } \\
\text { @ 193nm }\end{array}$ \\
\hline $\mathrm{Nb}-1$ & 1,970 & 191 & 0.38 \\
\hline $\mathrm{Nb}-2$ & 3,200 & 235 & 0.37 \\
\hline $\mathrm{Nb}-3$ & 3,900 & 249 & 0.34 \\
\hline
\end{tabular}

An important problem shown in Table 2 is the relatively high optical absorbance of these materials at $193 \mathrm{~nm}$. The free radical polymerized anhydride ring contributes to the high optical density, but the high optical densities and long absorbance tail found in these polymers (the optical absorbance at $248 \mathrm{~nm}$ is surprisingly high (ca. $0.15 / \mathrm{m}$ )) suggest that the rapid termination rate (discussed above) contributes to this unwanted absorbance. The low molecular weight of these polymers provides a high end group concentration. This absorbance may be due to vinyl groups at the chain ends of these polymers, as well as residual monomer contamination. Both influences would be at least partially addressed if the synthesis is adjusted to afford higher molecular weight polymers. It should be noted that other groups have achieved slightly higher molecular weights in these copolymerizations.[11]
The relatively high absorbance of anhydride polymers can also be partially explained by the intrinsic absorbance of the anhydride structure. Figure 3 shows the absorbance and film thickness change of a film of methacrylate terpolymer (3a) bearing carboxylic acid groups upon prolonged heating to generate the anhydride structure (3b). The absorbance of the film doubles (per micron) as the anhydride concentration in the polymer increases $(3 \mathrm{c})$.

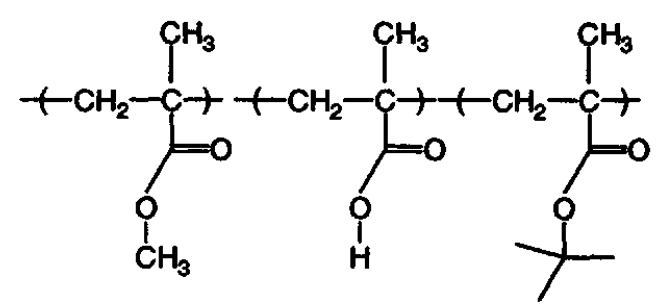

3(a)

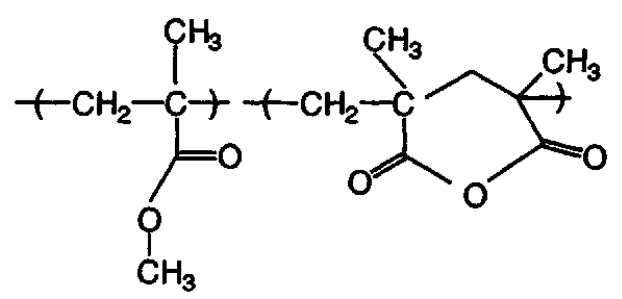

3(b)

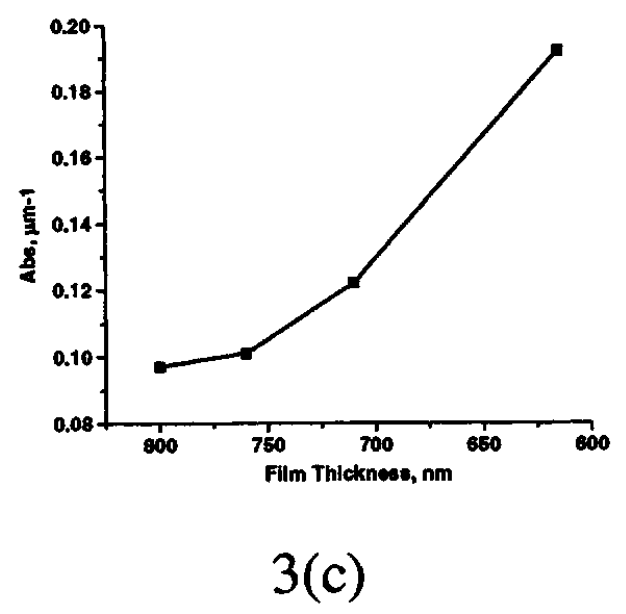

Figure 3a,b,c: Increased absorbance at $193 \mathrm{~nm}$ due to anhydride formation in a methacrylate polymer 

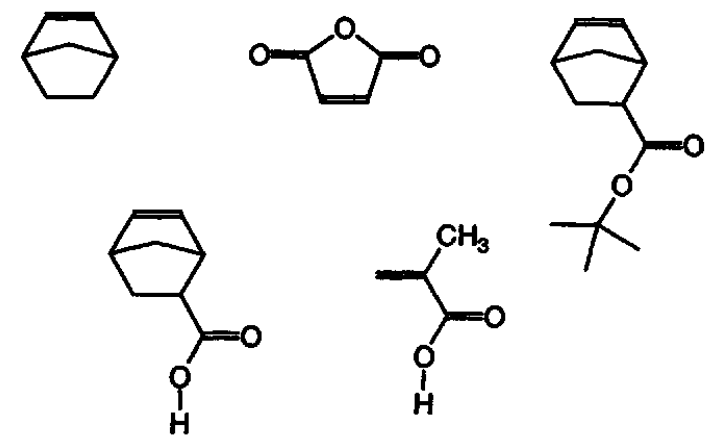

Figure 4: Molecular structures of monomers polymerized with maleic anhydride

We polymerized other (substituted) norbornene polymers with maleic anhydride. Figure 4 shows monomers that were used in this study. Hydroxylic monomers tend to increase yields, while monomers bearing bulky groups (active esters) tend to decrease yields.

A series of terpolymers were prepared which served as prototype resist polymers in this study. The terpolymerizations contained maleic anhydride (MAH)(50 mole percent) and a combination of norbornene $(\mathrm{Nb})$ and norbornene-t-butyl ester (Nb-t-BE) (Figure 5). The concentration of $\mathrm{Nb}-\mathrm{t}-\mathrm{BE}$ was varied from zero (poly(Nb/MAH)) to 50 mole percent (poly(Nb-t-BE/MAH)).

The glass transition temperature of these norbornene/norobornene ester/maleic anhydride terpolymers is not a strong function of monomer structure, although as shown above, it is dangerous to ignore molecular weight effects for this class of materials. Stated another way, the impact of a slight change in molecular weight likely masks (more subtle) side group effects in these alternating copolymers. The water contact angle, a measure of (surface) hydrophilicity, shows a clear trend in that apparently norbornene is a significantly more "polar" monomer than the t-butyl ester. These polymers were examined for carboxylic acid concentration by infrared spectroscopy, which revealed little or no anhydride ring opening. The high hydrophilicity of the norbornene/maleic anhydride polymer is substantially greater (as defined by low water contact angle) than other "193nm" polymers (acrylics and conventional cyclic olefins). This is particularly surprising since other polymers require 20-30 mole \% carboxylic acid to lower water contact angles to ca. 70 degrees. The high hydrophilicity of these polymers influences the unique dissolution properties of the alternating copolymers (see below).

The very simple norbornene/maleic anhydride/ester terpolymers provide relatively high resolution imaging without resorting to complex formulation and process development. Figure 6 shows contrast curve for one of these terpolymers of a $35 / 50 / 15$ compositions (15\% ester). Figure 7 shows images printed in a prototype formulation using the 25/50/25 terpolymer discussed above. It is noteworthy that these alternating polymer resists function admirably in strong developer and do not require carboxylic acid in the starting polymer.

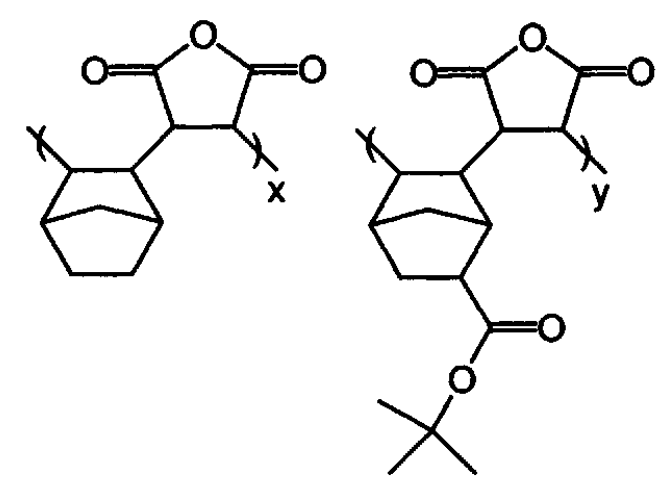

Figure 5: Chemical structure of prototype resist terpolymer

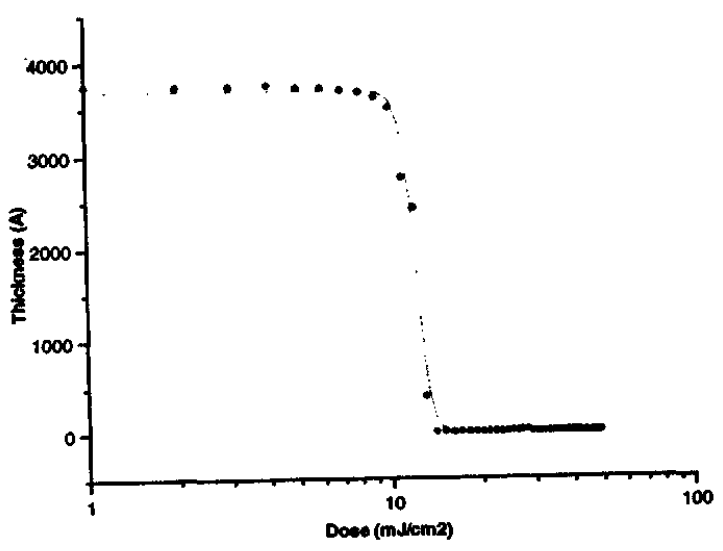

Figure 6: Contrast curve for a resist of $\mathrm{Nb} / \mathrm{MAH} / \mathrm{Nb}$-ester $(35 / 50 / 15)$ terpolymer 


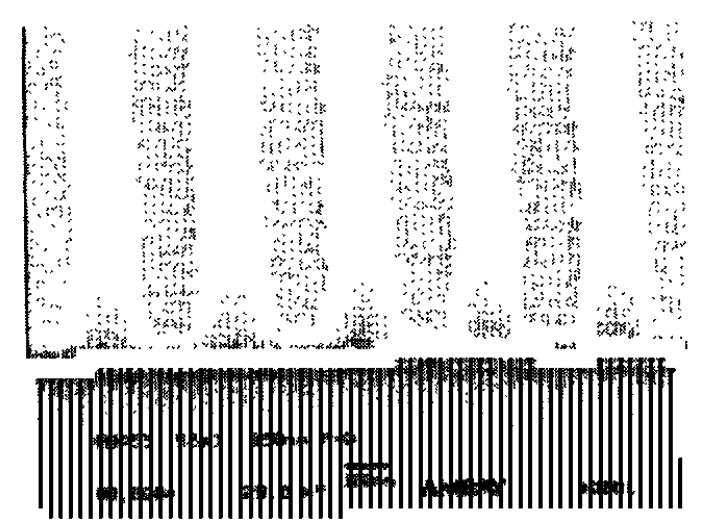

Figure 7: Fine features $(0.15 \mathrm{um})$ printed in $\mathrm{Nb} / \mathrm{MAH} / \mathrm{Nb}$-ester prototype resist formulation.

The surprising ease with which imaging and contrast properties were arrived at in these low protection level anhydride-containing polymers (with no carboxylic acid present in the starting material) suggested that the dissolution function of these resists was substantially different than in the other polymer families investigated. To probe these effects, a series of model deprotected polymers were prepared by copolymerizing norbornene/maleic anhydride and a third monomer representing a deprotected ester. Thus, two series of terpolymers of $\mathrm{Nb} / \mathrm{MAH}$ and norbornene-carboxylic acid ( $\mathrm{Nb}-\mathrm{COOH})$ (norbornene-t-butyl ester deprotection product) and $\mathrm{Nb} / \mathrm{MAH}$ and methacrylic acid (t-butyl methacrylate deprotection product) were prepared and their dissolution properties were examined. Figure 8 shows the dissolution properties as a function of carboxylic acid concentration as characterized by Quartz Crystal Microbalance measurements. The data clearly indicates why the resists with very low protecting group concentrations perform in this family of polymers. Even at ca. 10 mole percent carboxylic acid concentration, these materials develop in a straightforward linear fashion. The incorporation of ca. 20 mole percent carboxylic acid produces a rapid dissolution rate (ca. $5000 \mathrm{~A} / \mathrm{sec}$ ).

The etch resistance of cyclic olefin/maleic anhydride alternating copolymers in an oxide etch process in good, but not remarkable.
The simple copolymer of $\mathrm{Nb} / \mathrm{MAH}$ is substantially more robust than APEX in an oxide etch process (almost $20 \%$ improvement), but the $\mathrm{Nb}$-ester copolymer is quite similar to APEX in terms of etch rate. By minimization of protecting group concentration, intrinsic etch resistance improves, as well as lessening the impact of film thickness reduction through deprotection during etch. $[4,14]$

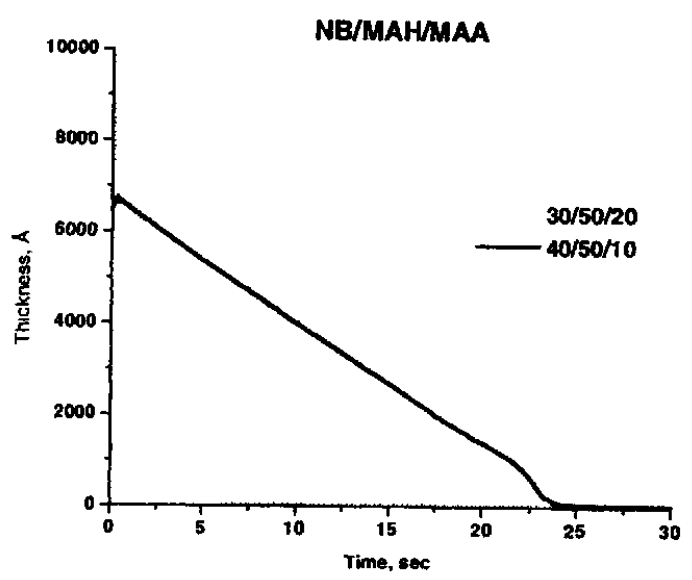

Figure 8: QCM dissolution curves for carboxylic acid-containing model polymers

\section{Summary}

The investigation of polymerization and properties of two classes of polymers for $193 \mathrm{~nm}$ resists has shown that each route is unique and has its own set of strengths and challenges. Methacrylate polymers are readily synthesized with tremendous control, can exhibit excellent resolution, but are difficult to completely control the resist dissolution properties and are quite limited with regard to oxide etch

resistance. Cyclic olefin/maleic anhydride alternating copolymers have some significant synthesis challenges with regard to control of the polymerization (moderate yields, poor molecular weight control) but are extremely tailorable in terms of resist dissolution properties and are surprisingly robust in terms of etch resistance, given the high oxygen content of maleic anhydride. The major limitation of these materials in our hands involves ramifications of the uncontrolled synthesis, leading to poor molecular 
weight control, extreme difficulty at reproducibly generating moderate molecular weights (above $5000 \mathrm{~g} / \mathrm{mole}$, approaching $10,000 \mathrm{~g} /$ mole), relatively high optical densities at $193 \mathrm{~nm}$ (and even at $248 \mathrm{~nm}$ ) and relatively low yields.

\section{Acknowledgments}

The authors thank Dr. Charles Wade of the IBM Almaden Research Center for his efforts in our NSF summer student program which contributed greatly to this project. Additionally, we thank Drs. S. Jayaraman and R. Vicari (BF Goodrich) for many useful, thought-provoking technical discussions.

\section{References}

1. R. D. Allen, W. E. Conley, and R. R. Kunz, "Deep-UV Resist Technology", Chp. 4 in Handbook of Microlithography, Ed. P. Rai-Choudhury, SPIE Press, 1997.

2. G. Wallraff, R. Allen, W. Hinsberg, L. Simpson, and R. Kunz, "Designing Tomorrow's Photoresists", Chemtech, April 1993, 22-30.

3. Y. Kaimoto, K. Nozaki, S. Takechi, N. Abe, Proc. SPIE, 1672, 66 (1992).

4. T. Wallow, P. Brock, J. Opitz, R. Allen, D. Hofer, Proc. SPIE, 3333, 92 (1998).

5. H. Ito, Jo. Photopolym. Sci. Technol, 11(3), 383 (1998).

6. T. Wallow, F. Houlihan, O. Nalamasu, E. Chandross, T. Neenan and E. Reichmanis, Proc. SPIE, 2724, 355 (1996).; U. Okoroanyanwu, T. Shimokawa, J. Byers, D. Medeiros, C. G. Willson, Q. Niu, J. Frechet, R. Allen, Proc. SPIE, 3049, 92 (1997); J. Jung, C. Bok, and K. Baik, Jo.
Photopolym. Sci. Technol., 10(4), 529 (1997).

7. R. Allen, R. Sooriyakumaran, J. Opitz, G. Wallraff, R. DiPietro, G. Breyta, D. Hofer, R. Kunz, S. Jayaraman, R. Shick, B. Goodall, U. Okoroanyanwu, C. G. Willson, Proc. SPIE, 2724, 334 (1996).; R. Allen, R. Sooriyakumaran, J. Opitz, G. Wallraff, R. DiPietro, G. Breyta, D. Hofer, R. Kunz, U. Okoroanyanwu, C. G. Willson, Jo. Photopolym. Sci. Technol, 9(3), 465 (1996); R. Allen, J. Opitz, T. Wallow, D. Hofer, S. Jayaraman, Proc. SPIE, 3333, 463 (1998).

8. U. Okoroanyanwu, T. Shimokawa, J. Byers, D. Medeiros, C. G. Willson, Q. Niu, J. Frechet, R. Allen, Proc. SPIE, 3049, 92 (1997); M. Suwa, T. Kajita, H. Iwasawa, M. Yamamoto, and $S$. Iwanaga, Proc. SPIE, 3333, 26 (1998). 9. Q. J. Niu, J.M.J. Frechet, U. Okoroanyanwu, J.D. Byers, and C.G. Willson, Proc. SPIE, 3049, 113 (1997); T. Hattori, Y. Tsuchiya, R. Yamanaka, K. Hattori, and H. Shiraishi, Jo. Photopolym. Sci. Technol., 10(4), 535 (1997).

10. R. Allen, I. Wan, G. Wallraff, R. DiPietro, D. Hofer and R. Kunz, Jo. Photopolym. Sci. Technol., 8(4), 623 (1995).

11. F. Houlihan, T. Wallow, O. Nalamasu, and E. eichmanis, Macromolecules, 30(21), 6517 (1997); U. Okoroanyanwu, T. Shimokawa, J. Byers, and C.G. Willson, Chem. Mater., 10(11), 3319 (1998). 12. G. Odian, Principles of Polymerization; Wiley: New York, 1981. 13. R. Allen, R. Chen and P. Wetmore, Proc. SPIE,2438, 250 (1995).

14. K. Patterson, U. Okoroanyanwu, $T$. Shimokawa, S. Cho, J. Byers, C. G. Willson, Proc. SPIE, 3333, 425 (1998). 